\title{
FLUCTUATIONS IN DOUBLY SCATTERED LASER LIGHT
}

\author{
F.C. VAN RIJSWIJK and U.L. SMITH \\ Fysisch Laboratorium Rijksuniversiteit Utrecht, The Netherlands
}

Received 25 September 1975

\begin{abstract}
Fluctuations in laser light, doubly scattered by brownian particles, were analysed by measuring the spectral noise power of the photodetector current. Scattering took place at two spatially separated systems of spherical particles. Analytic expressions for the field and intensity correlations are derived. The analytic expressions for the spectrum of the intensity fluctuations of the doubly scattered laser light demonstrate that the frequency dependence of the spectrum depends strongly on the geometry of the experimental arrangement. This is not the case for singly scattered light where in good approximation the spatial and temporal correlations can be separated analytically.

Our measurements show that the noise spectrum of the doubly scattered radiation may have the same frequency dependence as the spectrum of the singly scattered light. However, there are conditions where the frequency dependence of the noise of the doubly scattered light diverges markedly from that of the singly scattered light.
\end{abstract}

\section{Introduction}

Light beating spectroscopy of scattered laser light is a current technique used to investigate kinetic properties of the scattering medium ${ }^{1,2,3}$ ). Most studies on laser light scattering concern only single scattering. There are, however, scattering systems that are likely to produce double or even multiple scattering. At present it is not quite clear whether light beating spectroscopy in the case of double (or multiple) scattering can be a useful method in the study of the scattering medium. To solve this problem, however, a further theoretical and experimental study into the statistical properties of doubly scattered light is required.

We have investigated doubly scattered light in a very simple experimental set-up (see fig. 1). Scattering took place in two spatially separated cells each filled with the same dilute suspension of polystyrene spheres. A laser beam was focussed in one cell. The scattered light from this first cell was scattered for the second time in the second cell. The concentration of the scattering particles was so low that in each cell single scattering of the radiation dominated. A photomultiplier tube received only the radiation coming from the second cell. The particles in the cells undergo a brownian motion and the fluctuations in the light scattered by such a 
system of independent particles have been studied extensively ${ }^{1-14}$ ). In sec. 2 we shall give the field and intensity correlation functions of singly scattered light, referring to the literature.

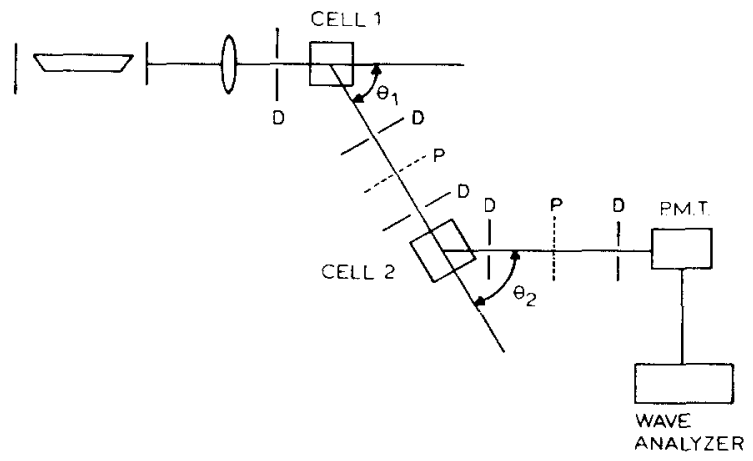

Fig. 1. Experimental set-up for the measurement of the noise spectral density of doubly scattered light. $D$ are diaphragms and $P$ are polarization analyzers. P.M.T. is the photomultiplier tube.

In the derivation of the correlation functions and spectral noise density of doubly scattered light, also given in sec. 2, we shall use certain assumptions that appeared to be satisfactory in the analysis of single scattering ${ }^{1-3}$ ). The incident laser beam is represented as a monochromatic plane wave with a constant amplitude across the scattering volume. Noise in the incident laser beam is neglected. The brownian particles are supposed to be dipole oscillators with dimensions less than the wavelength of the laser light (Rayleigh scattering) and the number of scatterers is assumed to be constant. Only experimental geometries are considered so that the direction of polarization of the scattered waves is nearly equal to that of the incident beam.

In sec. 3 the theoretical and experimental results for the noise spectral density of doubly scattered laser light (homodyne detection) are compared. In sec. 4 we shall discuss our theory and experiments and shall compare them with the results obtained by others ${ }^{15-17}$ ).

\section{Theory}

\subsection{Single scattering}

Let a linearly polarized plane monochromatic wave be singly scattered by a system of independent particles. For the cross-correlation of the scattered field (with same polarization as incident field) Tartaglia and $\mathrm{Chen}^{11}$ ) have derived 
that:

$$
\begin{aligned}
\Gamma_{1} & \left(\boldsymbol{R}_{\mathrm{P}}, \boldsymbol{R}_{\mathrm{Q}} ; \tau\right) \\
& \equiv\left\langle E_{1}^{*}\left(\boldsymbol{R}_{\mathrm{P}}, t\right) E_{1}\left(\boldsymbol{R}_{\mathrm{Q}}, t+\tau\right)\right\rangle \\
& =\left\langle E_{1}^{*}\left(\boldsymbol{R}_{\mathrm{P}}, t\right) E_{1}\left(\boldsymbol{R}_{\mathrm{Q}}, t\right)\right\rangle\left\langle E_{1}^{*}\left(\boldsymbol{R}_{\mathrm{P}}, t\right) E_{1}\left(\boldsymbol{R}_{\mathrm{P}}, t+\tau\right)\right\rangle\left\langle\left\langle I_{1}\left(\boldsymbol{R}_{\mathrm{P}}, t\right)\right\rangle,\right.
\end{aligned}
$$

where $\boldsymbol{R}_{\mathbf{P}}$ and $\boldsymbol{R}_{\mathbf{Q}}$ denote the position of $\mathrm{P}$ and $\mathrm{Q}$ respectively on the surface of a photodetector, $E_{1}\left(\boldsymbol{R}_{\mathrm{P}}, t\right)$ is the electric field strength at point $\mathrm{P}$ at time $t$ due to scattering by the particles and $I_{1}\left(\boldsymbol{R}_{\mathrm{P}}, t\right)$ is the intensity defined by:

$$
I_{\mathrm{t}}\left(\boldsymbol{R}_{\mathbf{P}}, t\right)=E_{1}^{*}\left(\boldsymbol{R}_{\mathbf{P}}, t\right) E_{1}\left(\boldsymbol{R}_{\mathbf{P}}, t\right) .
$$

The origin of the coordinate system is at the centre of the scattering cell. Note that in eq. (1) the spatial and the temporal correlations appear separately. For a system of $N$ independent spherical brownian particles the temperal correlation function has been found to $\mathrm{be}^{2,7}$ ):

$$
\begin{aligned}
& \left\langle E_{1}^{*}\left(\boldsymbol{R}_{\mathrm{P}}, t\right) E_{1}\left(\boldsymbol{R}_{\mathrm{P}}, t+\tau\right)\right\rangle \\
& \quad=\left(N \mu\left|E_{0}\right| / R_{0}\right)^{2} \exp \left(-\mathrm{i} \omega_{0} \tau-D\left|\boldsymbol{k}_{0}-\boldsymbol{k}_{\mathrm{P}}\right|^{2}|\tau|\right),
\end{aligned}
$$

where $E_{0}$ is the amplitude of the incident wave, which has an angular frequency $\omega_{0}$ and a wave vector in the medium $\boldsymbol{k}_{0}$. The wave vector of the scattered field along $\boldsymbol{R}_{\mathrm{P}}$ is denoted by $\boldsymbol{k}_{\mathrm{P}}$. Note that $k_{0}=k_{\mathrm{P}}=2 \pi n / \lambda$, where $n$ is the index of refraction of the medium and $\lambda$ is the wavelength in vacuum. The distance from the centre of the cell to the centre of the detector surface is $R_{0}$, the plane detector surface is supposed to be normal to $\boldsymbol{R}_{0} . D$ is the diffusion constant, which for spherical particles at temperature $T$ is ${ }^{18}$ ):

$$
D=k_{\mathrm{B}} T / 6 \pi \eta \rho
$$

Here $k_{\mathrm{B}}$ is Boltzmann's constant, $\eta$ is the viscosity of the solvent and $\varrho$ is the radius of the particle. In eq. (3) $\mu$ depends on wavelength and the polarizability of a scatterer $\left.^{19}\right)$. The explicit expression of $\mu$ is of no interest here.

If in eq. (3) we are allowed to put:

$$
\left|\boldsymbol{k}_{0}-\boldsymbol{k}_{\mathrm{P}}\right| \approx\left|\boldsymbol{k}_{0}-\boldsymbol{k}_{\mathrm{Q}}\right| \approx\left|\boldsymbol{k}_{0}-\boldsymbol{k}_{\mathrm{s}}\right|
$$

where $\boldsymbol{k}_{\mathrm{Q}}$ and $\boldsymbol{k}_{\mathrm{s}}$ are the wave vectors along the directions of $\boldsymbol{R}_{\mathbf{Q}}$ and $\boldsymbol{R}_{\mathbf{0}}$, respectively, the temporal coherence function in eq..(3) is independent of the position of the point $P$ on the detector surface. Eq. (5) generally holds if the linear dimensions of the detector surface are much less than the distance between detector and scattering cell. We should note that eq. (1) is only correct if eq. (5) holds ${ }^{11}$ ). 
The spatial coherence function $\left\langle E_{1}^{*}\left(\boldsymbol{R}_{\mathrm{P}}, t\right) E_{1}\left(\boldsymbol{R}_{\mathrm{Q}}, t\right)\right\rangle$ depends on the geometry of the scattering medium ${ }^{2,3,11}$ ) and in special cases expressions for it are given in the literature ${ }^{11,20}$ ). We shall not discuss this spatial coherence function here as we shall deal with spatial coherence functions in the derivation of doubly scattered light.

According to eq. (3):

$$
\left\langle I_{1}\right\rangle=\left\langle I_{1}\left(\boldsymbol{R}_{\mathrm{P}}, t\right)\right\rangle=\left\langle I_{1}\left(\boldsymbol{R}_{\mathrm{Q}}, t\right)\right\rangle=\left(N \mu\left|E_{0}\right| / R_{0}\right)^{2} .
$$

For the cross-correlation of the intensity of the single scattered field, defined by:

$$
\Phi_{1}\left(\boldsymbol{R}_{\mathbf{P}}, \boldsymbol{R}_{\mathbf{Q}} ; \tau\right)=\left\langle I_{1}\left(\boldsymbol{R}_{\mathbf{P}}, t\right) I_{1}\left(\boldsymbol{R}_{\mathbf{Q}}, t+\tau\right)\right\rangle,
$$

Tartaglia and Chen derived $\left.{ }^{11}\right)$ :

$$
\Phi_{1}\left(\boldsymbol{R}_{\mathbf{P}}, \boldsymbol{R}_{\mathrm{Q}} ; \tau\right)=\left\langle I_{1}\left(\boldsymbol{R}_{\mathbf{P}}, t\right)\right\rangle^{2}\left(1+\left|\Gamma_{1}\left(\boldsymbol{R}_{\mathbf{P}}, \boldsymbol{R}_{\mathrm{Q}} ; \tau\right)\right|^{2}\right)
$$

assuming that $N \gg 1$. Eq. (8) generally holds for gaussian fields ${ }^{21}$ ). From eqs. (1), (3) and (6) it now follows that:

$$
\begin{aligned}
\Phi_{1}\left(\boldsymbol{R}_{\mathrm{P}}, \boldsymbol{R}_{\mathrm{Q}} ; \tau\right)= & \left\langle I_{1}\right\rangle^{2}\left\{1+\exp \left(-2 D|\tau|\left|\boldsymbol{k}_{0}-\boldsymbol{k}_{\mathrm{s}}\right|^{2}\right)\right. \\
& \left.\times\left|\left\langle E_{1}^{*}\left(\boldsymbol{R}_{\mathrm{P}}, t\right) E_{1}\left(\boldsymbol{R}_{\mathrm{Q}}, t\right)\right\rangle\right|^{2}\right\}
\end{aligned}
$$

\subsection{Double scattering}

In fig. 1 an outline is given of the experimental set-up and the relevant vectors and distances in the double scattering experiment can be found in fig. 2 . The origin of the coordinate system is located at the centre $\mathrm{O}_{1}$ of the first cell. The centre of the second scattering cell $\mathrm{O}_{2}$ is at position $\boldsymbol{R}_{1}$ with respect to $\mathrm{O}_{1}$. The centre of the detector is at position $\boldsymbol{R}_{2}$, which vector has $\mathrm{O}_{2}$ as origin and is normal to the detector surface. The wave vector of the incident plane wave is denoted by $\boldsymbol{k}_{0} ; \boldsymbol{k}_{1}$ and $\boldsymbol{k}_{2}$ are wave vectors along $\boldsymbol{R}_{1}$ and $\boldsymbol{R}_{2}$, respectively. The first scattering angle $\theta_{1}$ is the angle between $\boldsymbol{k}_{0}$ and $\boldsymbol{k}_{1}$ and the second scattering angle $\theta_{2}$ is that between $\boldsymbol{k}_{1}$ and $\boldsymbol{k}_{2}$. The position of particle a (or b, c, d) in the first cell with respect to $\mathrm{O}_{1}$ is indicated by the vector $\boldsymbol{r}_{\mathrm{a}}$ (or $\boldsymbol{r}_{\mathrm{b}}, \boldsymbol{r}_{\mathrm{c}}, \boldsymbol{r}_{\mathrm{d}}$ ) whereas $\boldsymbol{s}_{\alpha}$ (or $\boldsymbol{s}_{\beta}, \boldsymbol{s}_{\gamma}, \boldsymbol{s}_{\delta}$ ) gives the position of particle $\alpha$ (or $\beta, \gamma, \delta$ ) in the second cell with respect to $\mathrm{O}_{2}$. The distance between a particle $a$ in the first and a particle $\alpha$ in the second cell is denoted by $r_{\mathrm{a} \alpha} . \mathrm{P}$ is a point at the detector surface. Its position with respect to $\mathrm{O}_{1}$ is indicated by the vector $\boldsymbol{R}_{\mathrm{P}}$ whereas $\boldsymbol{s}_{\mathrm{P}}$ gives the position of $\mathrm{P}$ with respect to $\mathrm{O}_{2}$. The distance between particle $\alpha$ belonging to the second cell and $\mathrm{P}$ is given by $r_{\alpha \mathrm{P}}$. 


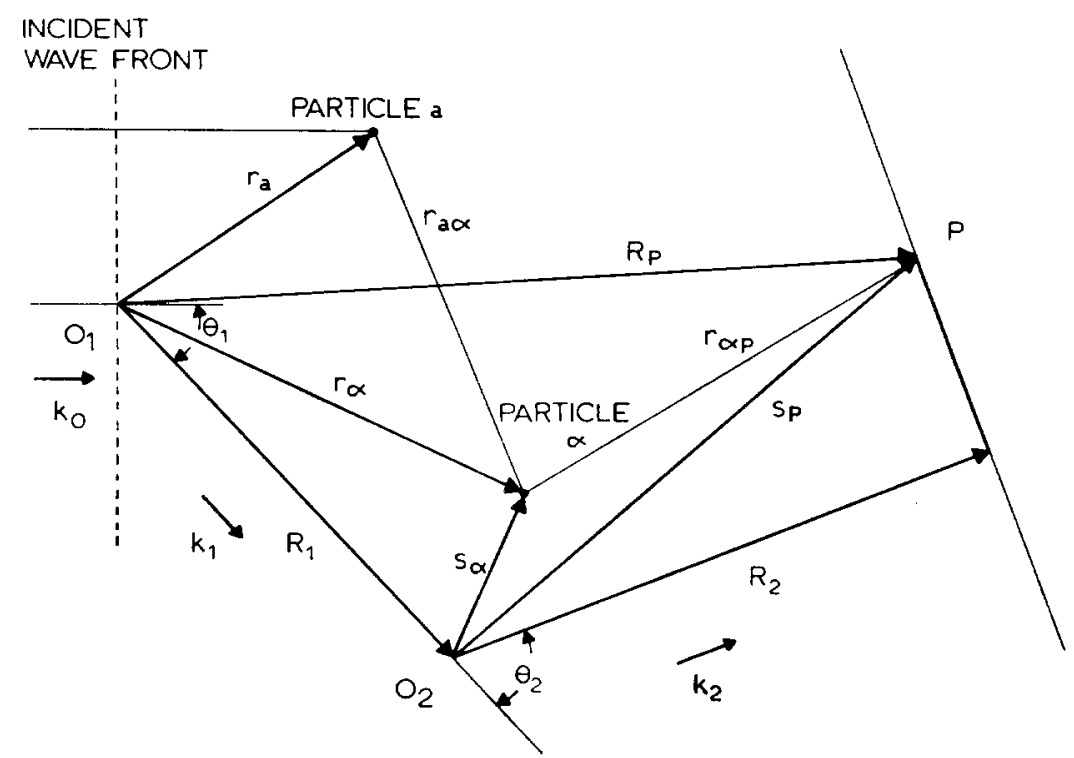

Fig. 2. Geometry for the calculation of the phase of light in point $P$ on the detector surface. The incident wave is scattered by particle a and subsequently by particle $\alpha$.

Let a linearly polarized plane wave of the form

$$
E(\boldsymbol{r}, t)=E_{0} \exp \left\{\mathbf{i}\left(\boldsymbol{k}_{0} \cdot \boldsymbol{r}-\omega_{0} t\right)\right\}
$$

be scattered by particles in the first cell. We shall consider only singly or doubly scattered waves, that propagate under an angle $\phi$ with respect to the direction of polarization of the initial plane wave with $\phi=90^{\circ} \pm 10 \%$. As a consequence the direction of polarization of the scattered waves is nearly that of the initial plane wave. The field at particle $\alpha$ in the second cell due to scattering by particle a in the first cell is:

$$
\left(\mu E_{0} / r_{\mathrm{a \alpha}}\right) \exp \left\{\mathrm{i}\left(\boldsymbol{k}_{\mathrm{o}} \cdot \boldsymbol{r}_{\mathbf{a}}+k_{\mathrm{o}} \boldsymbol{r}_{\mathrm{a} \alpha}-\omega_{0} t\right)\right\} .
$$

In eq. (11) the positions of all particles are taken at time $t$ as the displacement of the particles, defined by:

$$
\Delta r_{\mathrm{a}}=r_{\mathrm{a}}(t+\tau)-\boldsymbol{r}_{\mathrm{a}}(t)=\boldsymbol{r}_{\mathrm{a}}^{\prime}-\boldsymbol{r}_{\mathrm{a}}
$$

and

$$
\Delta s_{\alpha}=s_{\alpha}(t+\tau)-s_{\alpha}(t)=s_{\alpha}^{\prime}-s_{\alpha},
$$


is negligible with respect to $\lambda$ in time intervals of length $\tau=R_{1} / c$, where $c$ is the velocity of light in the medium. In the sequel we shall denote the position of a particle at time $t$ by $\boldsymbol{r}$ (or $s$ ) and the position of a particle at time $t+\tau$ by $\boldsymbol{r}^{\prime}$ (or $\boldsymbol{s}^{\prime}$ ).

The field at point $\mathrm{P}$ of a wave scattered by particle a and subsequently by particle $\alpha$ is :

$$
\left(\mu^{2} E_{0} / r_{a x} r_{\alpha \mathrm{P}}\right) \exp \left\{\mathrm{i}\left(\boldsymbol{k}_{0} \cdot \boldsymbol{r}_{a}+k_{0} r_{a \alpha}+k_{0} r_{\mathrm{aP}}-\sigma_{0} t\right)\right\} .
$$

Fluctuations in the total field at $\mathrm{P}$ will be primarily due to interference of waves with different phases. Therefore small differences in wave amplitude can be neglected since the linear dimensions of the scattering volumes are supposed to be small with respect to their distance $R_{1}$. So in the amplitude of the wave in eq. (13) we put:

$$
1 / r_{\mathrm{a} x} r_{\mathrm{aP}}=1 / R_{1} R_{2} .
$$

The total field at $\mathrm{P}$ at time $t$ due to double scattering is now found to be:

$$
\begin{aligned}
E_{2}\left(\boldsymbol{R}_{\mathbf{p}}, t\right)= & \left(\mu^{2} E_{0} / R_{1} R_{2}\right) \exp \left(-\mathrm{i} \omega_{0} t\right) \\
& \times \sum_{a=1}^{N_{1}} \sum_{i=1}^{N_{2}} \exp \left\{\mathrm{i}\left(\boldsymbol{k}_{0} \cdot \boldsymbol{r}_{a}+k_{0} r_{a \alpha}+k_{0} r_{\alpha \mathrm{P}}\right)\right\},
\end{aligned}
$$

where $N_{1}$ and $N_{2}$ are the total number of scatterers in cells 1 and 2, respectively.

Defining the intensity of the field at $\mathrm{P}$ by:

$$
I_{2}\left(\boldsymbol{R}_{\mathrm{P}}, t\right)=E_{2}^{*}\left(\boldsymbol{R}_{\mathrm{P}}, t\right) E_{2}\left(\boldsymbol{R}_{\mathrm{P}}, t\right),
$$

we obtain:

$$
\begin{aligned}
I_{2}\left(\boldsymbol{R}_{\mathrm{P}}, t\right)= & \left(\mu^{2}\left|E_{0}\right| / R_{1} R_{2}\right)^{2} \\
& \times \sum_{a, b}^{N_{1}} \sum_{\alpha, \beta}^{N_{2}} \exp \left[\mathrm{i}\left\{\boldsymbol{k}_{0} \cdot\left(\boldsymbol{r}_{b}-\boldsymbol{r}_{a}\right)+k_{0}\left(r_{b \beta}-r_{a \alpha}+r_{\beta \mathrm{P}}-r_{\alpha \mathrm{P}}\right)\right\}\right] .
\end{aligned}
$$

The positions of the particles are randomly distributed over the scattering volumes. The dimensions of the scattering volumes are supposed to be much greater than the wavelength. In the averaging over the positions of the particles only terms in eq. (17) with indices $a=b$ and $x=\beta$ are nonzero. Thus:

$$
\left\langle I_{2}\right\rangle=\left\langle I_{2}\left(\boldsymbol{R}_{\mathrm{P}}, t\right)\right\rangle=\left(\mu^{2}\left|E_{0}\right|^{2} / R_{1} R_{2}\right)^{2} N_{1} N_{2} .
$$


For the cross-correlation function of the field at $\mathrm{P}$ and $\mathrm{Q}$ we obtain with the help of eq. (15):

$$
\begin{aligned}
& \Gamma_{2}\left(\boldsymbol{R}_{\mathrm{P}}, \boldsymbol{R}_{\mathbf{Q}} ; \tau\right) \\
& \equiv\left\langle E_{2}^{*}\left(\boldsymbol{R}_{\mathbf{P}}, t\right) E_{2}\left(\boldsymbol{R}_{\mathbf{Q}}, t+\tau\right)\right\rangle=\left(\mu^{2}\left|E_{0}\right| / R_{1} R_{2}\right)^{2} \exp \left(-\mathrm{i} \omega_{0} \tau\right) \\
& \times\left\langle\sum_{a, b}^{N_{1}} \sum_{\alpha, \beta}^{N_{2}} \exp \left[\mathrm{i}\left\{\boldsymbol{k}_{\mathrm{O}} \cdot\left(\boldsymbol{r}_{b}^{\prime}-\boldsymbol{r}_{a}\right)+k_{0}\left(r_{b \beta}^{\prime}-r_{a_{\alpha}}+r_{\beta \mathrm{Q}}^{\prime}-r_{\alpha \mathrm{P}}\right)\right\}\right]\right\rangle .
\end{aligned}
$$

And for the cross-correlation of the intensity at points $\mathrm{P}$ and $\mathrm{Q}$ we find:

$$
\begin{aligned}
\Phi_{2}\left(\boldsymbol{R}_{\mathrm{P}}, \boldsymbol{R}_{\mathrm{Q}} ; \tau\right) \equiv & \left\langle I_{2}\left(\boldsymbol{R}_{\mathrm{P}}, t\right) I_{2}\left(\boldsymbol{R}_{\mathrm{Q}}, t+\tau\right)\right\rangle \\
= & \left(\mu^{2}\left|E_{0}\right| / R_{1} R_{2}\right)^{4}\left\langle\sum _ { a , b , c , d } ^ { N _ { 1 } } \sum _ { \alpha , \beta , \gamma , \delta } ^ { N _ { 2 } } \operatorname { e x p } \left[\mathrm { i } \left\{ k_{0} \cdot\left(\boldsymbol{r}_{b}-\boldsymbol{r}_{a}+\boldsymbol{r}_{d}^{\prime}-\boldsymbol{r}_{c}^{\prime}\right)\right.\right.\right. \\
& \left.\left.\left.+k_{\mathrm{o}}\left(r_{b \beta}-r_{a \alpha}+r_{\mathrm{d} \delta}^{\prime}-r_{c \gamma}^{\prime}+r_{\beta \mathrm{P}}-r_{\alpha \mathrm{P}}+r_{\delta \mathrm{Q}}^{\prime}-r_{\gamma \mathrm{Q}}^{\prime}\right)\right\}\right]\right\rangle \cdot \quad(20)
\end{aligned}
$$

In general the ensemble averaging in eqs. (19) and (20) does not lead to amenable expressions. Therefore we shall make the following approximations. First we suppose the dimensions of the second scattering volume to be much less than $R_{2}$. So:

$$
r_{\alpha \mathbf{P}}=\left|s_{\mathbf{P}}-s_{\alpha}\right| \simeq s_{\mathbf{P}}-s_{\alpha} \cdot \hat{s}_{\mathbf{P}},
$$

where $\hat{\boldsymbol{s}}_{\mathrm{p}}$ is a unit vector in the direction $\boldsymbol{s}_{\mathrm{p}}$. If we use the notation $\boldsymbol{k}_{\mathrm{P}}=k_{0} \hat{s}_{\mathrm{P}}$ and $\boldsymbol{k}_{\mathrm{Q}}=k_{0} \hat{s}_{\mathrm{Q}}$ we obtain with the help of eq. (21):

$$
\begin{aligned}
I_{2} & \left(\boldsymbol{R}_{\mathrm{P}}, \boldsymbol{R}_{\mathrm{Q}} ; \tau\right) \\
= & \left(\mu^{2}\left|E_{0}\right| / R_{1} R_{2}\right)^{2} \exp \left\{-\mathrm{i} \omega_{0} \tau+\mathrm{i} k_{0}\left(s_{\mathrm{Q}}-s_{\mathrm{P}}\right)\right\} \\
& \times\left\langle\sum_{a, b}^{N_{1}} \sum_{\alpha, \beta}^{N_{2}} \exp \left[\mathrm{i}\left\{\boldsymbol{k}_{0} \cdot\left(\boldsymbol{r}_{b}^{\prime}-\boldsymbol{r}_{a}\right)+k_{0}\left(r_{b \beta}^{\prime}-r_{a \alpha}\right)-\left(\boldsymbol{k}_{\mathrm{O}} \cdot \boldsymbol{s}_{\beta}^{\prime}-\boldsymbol{k}_{\mathrm{P}} \cdot \boldsymbol{s}_{\alpha}\right)\right\}\right]\right\rangle,
\end{aligned}
$$

and

$$
\begin{aligned}
\Phi_{2} & \left(\boldsymbol{R}_{\mathrm{P}}, \boldsymbol{R}_{\mathrm{Q}} ; \tau\right) \\
= & \left(\mu^{2}\left|E_{0}\right| / R_{1} R_{2}\right)^{4}\left\langle\sum _ { a , b , c , d } ^ { N _ { 1 } } \sum _ { \alpha , \beta , \gamma , \delta } ^ { N _ { 2 } } \operatorname { e x p } \left[\mathrm { i } \left\{\boldsymbol{k}_{0} \cdot\left(\boldsymbol{r}_{b}-\boldsymbol{r}_{a}+\boldsymbol{r}_{d}^{\prime}-\boldsymbol{r}_{c}^{\prime}\right)\right.\right.\right. \\
& \left.\left.\left.+k_{0}\left(r_{b \beta}-r_{a \alpha}+r_{d \delta}^{\prime}-r_{c \gamma}^{\prime}\right)-\boldsymbol{k}_{\mathrm{P}} \cdot\left(\boldsymbol{s}_{\beta}-\boldsymbol{s}_{\alpha}\right)-\boldsymbol{k}_{\mathrm{Q}} \cdot\left(\boldsymbol{s}_{\delta}^{\prime}-\boldsymbol{s}_{\gamma}^{\prime}\right)\right\}\right]\right\rangle .
\end{aligned}
$$


Next we distinguish two cases:

(i) the cells are close together but the linear dimensions of the scattering volumes are less than $R_{1}$ by about a factor 10 ;

(ii) the cells are far apart. Now the linear dimensions of the scattering volumes are supposed to be much smaller than $R_{1}$. Thus:

$$
r_{a x}=\left|\boldsymbol{r}_{a}-\boldsymbol{r}_{a}\right| \simeq r_{a}-\boldsymbol{r}_{a} \cdot \hat{r}_{a} .
$$

We shall calculate the field and intensity correlations for both cases separately.

\subsection{Case (i), cells close together}

The ensemble averaging in eq. (22) over the random positions of the particles cannot be carried out separately over both scattering volumes. Because of the statistical independence of the positions of the particles only terms in eq. (22) with indices $a=b$ and $\alpha=\beta$ are nonzero. The displacements $A \boldsymbol{r}_{a}$ and $A \boldsymbol{s}_{\alpha}$ in a time-interval $\tau \simeq t_{\mathrm{c}}$, where $t_{\mathrm{c}}$ is the correlation time of the singly scattered field, are of the order of the magnitude of the wavelength $\lambda$ of the laser light (see appendix). So we can use the following approximation:

$$
r_{a x}^{\prime}=\left|R_{1}+s_{x}^{\prime}-r_{a}^{\prime}\right|=r_{a \alpha}+\left(\Delta s_{\alpha}-\Delta r_{a}\right) \cdot \hat{r}_{a x},
$$

where $\hat{r}_{a \alpha}$ is a unit vector along $r_{a \alpha}$ in the direction of particle $\alpha$. Eq. (22) now becomes:

$$
\begin{aligned}
\Gamma_{2} & \left(\boldsymbol{R}_{\mathrm{P}}, \boldsymbol{R}_{\mathrm{Q}} ; \tau\right) \\
= & N_{1} N_{2}\left(\mu^{2}\left|E_{0}\right| / R_{1} R_{2}\right)^{2} \exp \left\{-\mathrm{i} \omega_{0} \tau+\mathrm{i} k_{\mathrm{O}}\left(s_{\mathrm{Q}}-s_{\mathrm{P}}\right)\right\} \\
& \times\left\langle\exp \left[\mathrm{i}\left\{\left(\boldsymbol{k}_{0}-\boldsymbol{k}_{a x}\right) \cdot \mid \boldsymbol{r}_{a}+\left(\boldsymbol{k}_{a x}-\boldsymbol{k}_{\mathrm{Q}}\right) \cdot \Delta \boldsymbol{s}_{\mathrm{x}}-\left(\boldsymbol{k}_{\mathrm{Q}}-\boldsymbol{k}_{\mathrm{P}}\right) \cdot \boldsymbol{s}_{x}\right\}\right]\right\rangle,
\end{aligned}
$$

where $\boldsymbol{k}_{a x}=k_{0} \hat{r}_{a x}$. Note that in eq. (26) we must average over particle positions at $t$ and over the displacement of a particle in a time-interval of length $\tau$. As is well known from the theory of brownian motion the probability of occurrence of a particle at $\boldsymbol{r}+\Delta \boldsymbol{r}$ at time $t+\tau$ when it was at $\boldsymbol{r}$ at time $t$ is given by ${ }^{22}$ ):

$$
P(\boldsymbol{r}+\Delta \boldsymbol{r} \mid \boldsymbol{r})=(4 \pi D \tau)^{-3 / 2} \exp \left(-(\Delta r)^{2} / 4 D \tau\right)
$$

Carrying out the ensemble averaging over $\Delta \boldsymbol{r}_{a}$ and $\Delta \boldsymbol{s}_{\alpha}$ in eq. (26) with the help of eq. (27) we obtain:

$$
\begin{aligned}
\Gamma_{2} & \left(\boldsymbol{R}_{\mathrm{P}}, \boldsymbol{R}_{\mathrm{Q}} ; \tau\right) \\
= & N_{1} N_{2}\left(\mu^{2}\left|E_{0}\right| / R_{1} R_{2}\right)^{2} \exp \left\{-\mathrm{i} \omega_{0} \tau+\mathrm{i} k_{0}\left(s_{\mathrm{Q}}-s_{\mathrm{P}}\right)\right\} \\
& \quad \times\left\langle\exp \left\{-D|\tau|\left(\left|\boldsymbol{k}_{0}-\boldsymbol{k}_{a \alpha}\right|^{2}+\left|\boldsymbol{k}_{\boldsymbol{a} x}-\boldsymbol{k}_{\mathrm{Q}}\right|^{2}\right)+\mathrm{i}\left(\boldsymbol{k}_{\mathrm{P}}-\boldsymbol{k}_{\mathrm{Q}}\right) \cdot \boldsymbol{s}_{\alpha}\right\}\right\rangle .
\end{aligned}
$$


In eq. (28) ensemble averaging still has to be performed over the statistical positions of the scatterers $a$ and $\alpha$. Note that even if:

$$
\left|\boldsymbol{k}_{a x}-\boldsymbol{k}_{\mathrm{Q}}\right| \simeq\left|\boldsymbol{k}_{a x}-\boldsymbol{k}_{2}\right|,
$$

the spatial and the temporal correlations in $\Gamma_{2}\left(\boldsymbol{R}_{\mathrm{P}}, \boldsymbol{R}_{\mathrm{Q}} ; \tau\right)$ cannot be separated (cross-spectrally impure $\left.{ }^{23}\right)$ ). But if we are allowed to assume that the factor between ensemble brackets in eq. (28) is about equal to:

$$
\begin{aligned}
\langle\cdots\rangle \simeq & \left\langle\exp \left\{\mathrm{i}\left(\boldsymbol{k}_{\mathrm{P}}-\boldsymbol{k}_{\mathrm{Q}}\right) \cdot \boldsymbol{s}_{\alpha}\right\}\right\rangle \\
& \times \exp \left\{-D|\boldsymbol{\tau}|\left(\left|\boldsymbol{k}_{0}-\boldsymbol{k}_{1}\right|^{2}+\left|\boldsymbol{k}_{1}-\boldsymbol{k}_{2}\right|^{2}\right)\right\},
\end{aligned}
$$

separation of temporal from spatial correlation is obtained:

$$
\begin{aligned}
I_{2}\left(\boldsymbol{R}_{\mathbf{P}}, \boldsymbol{R}_{\mathrm{Q}} ; \tau\right)= & N_{1} N_{2}\left(\mu^{2}\left|E_{0}\right| / R_{1} R_{2}\right)^{2} \\
& \times \exp \left\{\mathrm{i} k_{0}\left(s_{\mathrm{Q}}-s_{\mathrm{P}}\right)\right\}\left\langle\exp \left\{\mathrm{i}\left(\boldsymbol{k}_{\mathrm{P}}-\boldsymbol{k}_{\mathrm{Q}}\right) \cdot \boldsymbol{s}_{\alpha}\right\}\right\rangle \\
& \times \exp \left\{-\mathrm{i} \omega_{0} \tau-D|\tau|\left(\left|\boldsymbol{k}_{0}-\boldsymbol{k}_{1}\right|^{2}+\left|\boldsymbol{k}_{1}-\boldsymbol{k}_{2}\right|^{2}\right)\right\} .
\end{aligned}
$$

Whether eq. (30) holds or not depends on the dimensions of both scattering volumes. But if $\left(\left|\boldsymbol{k}_{0}-\boldsymbol{k}_{a x}\right|^{2}+\left|\boldsymbol{k}_{a \alpha}-\boldsymbol{k}_{\mathrm{Q}}\right|^{2}\right)$ can be approximated by $\left(\left|\boldsymbol{k}_{0}-\boldsymbol{k}_{1}\right|^{2}\right.$ $+\left|\boldsymbol{k}_{1}-\boldsymbol{k}_{2}\right|^{2}$ ) within $1 \%$ for all values of the index $a \alpha$, eq. (30) can be accepted.

Next we shall calculate the cross-correlation of the light intensity. Only terms in eq. (23) with indices:

$$
a=b, \quad c=d \quad \text { and } \quad \alpha=\beta, \quad \gamma=\delta
$$

or

$$
a=d, \quad b=c, \quad a \neq b \quad \text { and } \quad \alpha=\delta, \quad \beta=\gamma, \quad \alpha \neq \beta
$$

are nonzero after ensemble averaging. Therefore we can write:

$$
\begin{aligned}
\Phi_{2}\left(\boldsymbol{R}_{\mathrm{P}}, \boldsymbol{R}_{\mathrm{Q}} ; \tau\right)= & \left(\mu^{2}\left|E_{0}\right| / R_{1} R_{2}\right)^{4}\left[N_{1}^{2} N_{2}^{2}\right. \\
& +\sum_{\substack{a, b \\
a \neq b}}^{N_{1}} \sum_{\substack{\alpha \neq \beta \\
\alpha \neq \beta}}^{n_{2}}\left\langle\operatorname { e x p } \left[\mathrm { i } \left\{\left(\boldsymbol{k}_{\mathrm{Q}}-\boldsymbol{k}_{a \alpha}\right) \cdot \Delta \boldsymbol{r}_{a}-\left(\boldsymbol{k}_{0}-\boldsymbol{k}_{b \beta}\right) \cdot \Delta \boldsymbol{r}_{b}\right.\right.\right. \\
& +\left(\boldsymbol{k}_{a \alpha}-\boldsymbol{k}_{\mathrm{Q}}\right) \cdot \Delta \boldsymbol{s}_{\alpha}-\left(\boldsymbol{k}_{b \beta}-\boldsymbol{k}_{\mathrm{Q}}\right) \cdot \Delta \boldsymbol{s}_{p} \\
& \left.\left.\left.\left.+\left(\boldsymbol{k}_{\mathrm{P}}-\boldsymbol{k}_{\mathrm{Q}}\right) \cdot\left(\boldsymbol{s}_{\alpha}-\boldsymbol{s}_{\beta}\right)\right\}\right]\right\rangle\right] .
\end{aligned}
$$

In the derivation of eq. (33) from eq. (23) we have used eq. (25). Working out the averaging over $\Delta \boldsymbol{r}_{a}, \Delta \boldsymbol{r}_{b}, \Delta \boldsymbol{s}_{\alpha}$ and $\Delta \boldsymbol{s}_{\beta}$ and assuming that $N_{1} \gg 1$ and $N_{2} \gg 1$, we 
obtain :

$$
\begin{aligned}
\Phi_{2}\left(\boldsymbol{R}_{\mathbf{P}}, \boldsymbol{R}_{\mathrm{Q}} ; \tau\right)= & \left(N_{1} N_{2}\right)^{2}\left(\mu^{2}\left|E_{6}\right| \mid R_{1} R_{2}\right)^{4} \\
& \times\left[1+\mid\left\langle\operatorname { e x p } \left\{-D|\tau|\left(\left|\boldsymbol{k}_{\mathrm{O}}-\boldsymbol{k}_{a,}\right|^{2}+\left|\boldsymbol{k}_{a,}-\boldsymbol{k}_{\mathrm{O}}\right|^{2}\right)\right.\right.\right. \\
& \left.\left.\left.+\mathrm{i}\left(\boldsymbol{k}_{\mathrm{P}}-\boldsymbol{k}_{\mathrm{Q}}\right) \cdot \boldsymbol{s}_{x}\right\}\right\rangle\left.\right|^{2}\right] .
\end{aligned}
$$

In eq. (34) the ensemble averaging has to be taken over the positions of one particle $a$ and one particle $x$. Spatial and temporal correlations in the intensity at the detector surface appear to be connnected. However, if eq. (30) holds, we find:

$$
\begin{aligned}
\Phi_{2} & \left(\boldsymbol{R}_{\mathrm{P}}, \boldsymbol{R}_{\mathrm{Q}} ; \tau\right) \\
= & \left(N_{1} N_{2}\right)^{2}\left(\mu^{2}\left|E_{0}\right| / \boldsymbol{R}_{1} R_{2}\right)^{2}\left|\left\langle\exp \left\{\mathrm{i}\left(\boldsymbol{k}_{\mathrm{P}}-\boldsymbol{k}_{\mathrm{O}}\right) \cdot \boldsymbol{s}_{\lambda}\right\}\right\rangle\right|^{2} \\
& \times\left[1+\exp \left\{-2 D|\tau|\left(\left|\boldsymbol{k}_{0}-\boldsymbol{k}_{1}\right|^{2}+\left|\boldsymbol{k}_{1}-\boldsymbol{k}_{2}\right|^{2}\right)^{2}\right] .\right.
\end{aligned}
$$

By comparison of eq. (34) with eq. (28) or by comparison of eq. (35) with eq. (31) it can be seen that:

$$
\Phi_{2}\left(\boldsymbol{R}_{\mathrm{P}}, \boldsymbol{R}_{\mathrm{Q}} ; \tau\right)=\left\langle I_{2}\right\rangle\left[1+\left|I_{2}\left(\boldsymbol{R}_{\mathrm{P}}, \boldsymbol{R}_{\mathrm{Q}}: \tau\right)\right|^{2}\right] .
$$

We note that for singly scattered light an equation equivalent to eq. (36) holds.

\subsection{Case (ii), cells far apart}

Noting that $r_{a} \ll r_{x}$ we find in addition to eq. (24):

$$
r_{a, 2}^{\prime} \simeq r_{x}^{\prime}-r_{a}^{\prime} \cdot \hat{r}_{a}^{\prime} \simeq r_{x}^{\prime}-\frac{r_{a}^{\prime} \cdot\left(r_{x}+1 s_{x}\right)}{r_{x}+1 s_{3} \cdot \hat{r}_{1}} \simeq r_{\alpha}^{\prime}-r_{a}^{\prime} \cdot \hat{r}_{\alpha},
$$

where

$$
r_{a}^{\prime} \simeq r_{a}+1 s_{\imath} \cdot \hat{\boldsymbol{r}}_{\mathrm{a}} .
$$

By the use of eqs. (24) and (37) we can write for the cross-correlation of the optical field given in eq. (22):

$$
\begin{aligned}
& \Gamma_{2}\left(\boldsymbol{R}_{\mathrm{P}}, \boldsymbol{R}_{\mathrm{Q}} ; \tau\right) \\
& =\left(\mu^{2}\left|E_{0}\right| / R_{1} R_{2}\right)^{2} \exp \left\{-\mathrm{i}\left(\prime_{0} \tau+\mathrm{i} k_{0}\left(s_{\mathrm{Q}}-s_{\mathrm{P}}\right)\right\}\right. \\
& \times\left\langle\sum _ { a , b } ^ { N _ { 1 } } \sum _ { i , \beta } ^ { N _ { 2 } } \operatorname { e x p } \left[\mathrm { i } \left\{\left(\boldsymbol{k}_{0}-\boldsymbol{k}_{\beta}\right) \cdot\left(\boldsymbol{r}_{b}+1 \boldsymbol{r}_{b}\right)-\left(\boldsymbol{k}_{0}-\boldsymbol{k}_{\imath}\right) \cdot \boldsymbol{r}_{a}\right.\right.\right. \\
& \left.\left.\left.+k_{0}\left(r_{\beta}-r_{\wedge}\right)+\left(\boldsymbol{k}_{\beta}-\boldsymbol{k}_{\mathrm{Q}}\right) \cdot \mid s_{\beta}-\left(\boldsymbol{k}_{\mathrm{Q}} \cdot \boldsymbol{s}_{\beta}-\boldsymbol{k}_{\mathrm{P}} \cdot \boldsymbol{s}_{\imath}\right)\right\}\right]\right\rangle \text {, }
\end{aligned}
$$


where $k_{\alpha}=k_{0} \hat{\boldsymbol{r}}_{\alpha}$ and $\boldsymbol{k}_{\beta}=k_{0} \hat{\boldsymbol{r}}_{\beta}$. Only terms with indices $b=a$ and $\alpha=\beta$ in eq. (38) give nonzero contributions. After the averaging over the displacements $\Delta \boldsymbol{r}_{a}$ and $\Delta \boldsymbol{s}_{\boldsymbol{\beta}}$ and over $\boldsymbol{r}_{a}$, which is homogeneously distributed over the first scattering volume, eq. (38) yields:

$$
\begin{aligned}
& \Gamma_{2}\left(\boldsymbol{R}_{\mathrm{P}}, \boldsymbol{R}_{\mathrm{Q}} ; \tau\right) \\
& =N_{1} N_{2}\left(\mu^{2}\left|E_{0}\right| / R_{1} R_{2}\right)^{2} \exp \left\{-\mathrm{i} \omega_{0} \tau+\mathrm{i} k_{0}\left(s_{\mathrm{Q}}-s_{\mathrm{P}}\right)\right\} \\
& \quad \times\left\langle\exp \left\{-D|\tau|\left(\left|\boldsymbol{k}_{0}-\boldsymbol{k}_{\alpha}\right|^{2}+\left|\boldsymbol{k}_{\alpha}-\boldsymbol{k}_{\mathrm{Q}}\right|^{2}\right)+\mathrm{i}\left(\boldsymbol{k}_{\mathrm{P}}-\boldsymbol{k}_{\mathrm{Q}}\right) \cdot \boldsymbol{s}_{\alpha}\right\}\right\rangle .
\end{aligned}
$$

If the linear dimensions of the second scattering volume are (much) smaller than $R_{1}$ and $R_{2}$ and if the linear dimension of the detector surface is small compared to $R_{2}$ we have:

$$
\left|\boldsymbol{k}_{0}-\boldsymbol{k}_{\alpha}\right|^{2}+\left|\boldsymbol{k}_{x}-\boldsymbol{k}_{\mathrm{Q}}\right|^{2} \simeq\left|\boldsymbol{k}_{0}-\boldsymbol{k}_{1}\right|^{2}+\left|\boldsymbol{k}_{1}-\boldsymbol{k}_{2}\right|^{2} .
$$

So then we find the final result:

$$
\begin{aligned}
\Gamma_{2}\left(\boldsymbol{R}_{\mathrm{P}}, \boldsymbol{R}_{\mathbf{Q}} ; \tau\right)= & N_{1} N_{2}\left(\mu^{2}\left|E_{0}\right| / R_{1} R_{2}\right)^{2} \\
& \times \exp \left\{\mathrm{i} k_{0}\left(s_{\mathrm{Q}}-s_{\mathrm{P}}\right)\right\}\left\langle\exp \left\{\mathrm{i}\left(\boldsymbol{k}_{\mathrm{P}}-\boldsymbol{k}_{\mathrm{Q}}\right) \cdot \boldsymbol{s}_{\alpha}\right\}\right\rangle \\
& \times \exp \left\{-\mathrm{i} \omega_{0} \tau-D|\tau|\left(\left|\boldsymbol{k}_{0}-\boldsymbol{k}_{1}\right|^{2}+\left|\boldsymbol{k}_{1}-\boldsymbol{k}_{2}\right|^{2}\right)\right\} .
\end{aligned}
$$

Note that eq. (41) equals eq. (31).

With the help of eqs. (23), (24) and (37) we can write for the cross-correlation function of the intensity:

$$
\begin{aligned}
\Phi_{2} & \left(\boldsymbol{R}_{\mathrm{P}}, \boldsymbol{R}_{\mathrm{Q}} ; \tau\right) \\
= & \left(\mu^{2}\left|E_{0}\right| / \boldsymbol{R}_{1} R_{2}\right)^{4}\left\langle\sum _ { a , b , c , d } ^ { N _ { 1 } } \sum _ { \alpha , \beta , \gamma , \delta } ^ { N _ { 2 } } \operatorname { e x p } \left[\mathrm { i } \left\{ k_{0} \cdot\left(\boldsymbol{r}_{b}-\boldsymbol{r}_{a}+\boldsymbol{r}_{d}^{\prime}-\boldsymbol{r}_{c}^{\prime}\right)\right.\right.\right. \\
& +k_{0}\left(r_{\beta}-r_{\alpha}+r_{\delta}^{\prime}-r_{\gamma}^{\prime}\right)-k_{0}\left(\boldsymbol{r}_{b} \cdot \hat{\boldsymbol{r}}_{\beta}-\boldsymbol{r}_{a} \cdot \hat{\boldsymbol{r}}_{\alpha}+\boldsymbol{r}_{d}^{\prime} \cdot \hat{\boldsymbol{r}}_{\delta}-\boldsymbol{r}_{c}^{\prime} \cdot \hat{\boldsymbol{r}}_{\gamma}\right) \\
& \left.\left.\left.\quad-\boldsymbol{k}_{\mathrm{P}} \cdot\left(\boldsymbol{s}_{\beta}-\boldsymbol{s}_{\alpha}\right)-\boldsymbol{k}_{\mathrm{Q}} \cdot\left(\boldsymbol{s}_{\delta}^{\prime}-\boldsymbol{s}_{\gamma}^{\prime}\right)\right\}\right]\right\rangle .
\end{aligned}
$$

Carrying out the ensemble averaging in eq. (42) we deal only with terms with indices:
(1) $a=b, c=d$,
(I) $\alpha=\beta, \gamma=\delta$,
(2) $a=d, \quad b=c, a \neq b$,
(II) $\alpha=\delta, \quad \beta=\gamma, \alpha \neq \beta$. 
Other terms are zero in a trivial way. Combination of the cases (1) and (2) with the cases (I) and (II) gives four cases in all. If we neglect the amplitude factor in eq. (42) we find after some arithmetic:

$$
\begin{aligned}
(1 \mathrm{I})= & N_{1}^{2} N_{2}^{2}, \\
(2 \mathrm{I})= & \left\langle\sum_{a, b}^{N_{1}} \sum_{x, y}^{N_{2}} \exp \left[\mathrm{i}\left\{\left(\boldsymbol{k}_{\mathrm{o}}-\boldsymbol{k}_{\gamma}\right) \cdot\left(\Delta \boldsymbol{r}_{a}-\Delta \boldsymbol{r}_{b}\right)+\left(\boldsymbol{k}_{\alpha}-\boldsymbol{k}_{\gamma}\right) \cdot\left(\boldsymbol{r}_{a}-\boldsymbol{r}_{b}\right)\right\}\right]\right\rangle \\
= & N_{1}\left(N_{1}-1\right) N_{2}^{2}\left\langle\exp \left\{\mathrm{i}\left(\boldsymbol{k}_{x}-\boldsymbol{k}_{\gamma}\right) \cdot\left(\boldsymbol{r}_{a}-\boldsymbol{r}_{b}\right)\right\} \exp \left(-2 D|\tau|\left|\boldsymbol{k}_{0}-\boldsymbol{k}_{i}\right|^{2}\right)\right\rangle, \\
(\mathrm{III})= & \left\langle\sum _ { a , c } ^ { N _ { 1 } } \sum _ { \alpha , \beta } ^ { N _ { 2 } } \operatorname { e x p } \left[\mathrm { i } \left\{\left(\boldsymbol{k}_{\alpha}-\boldsymbol{k}_{\mathrm{O}}\right) \cdot \Delta \boldsymbol{s}_{x}-\left(\boldsymbol{k}_{\beta}-\boldsymbol{k}_{\mathrm{O}}\right) \cdot \Delta s_{\beta}\right.\right.\right. \\
& \left.\left.\left.+\left(\boldsymbol{k}_{x}-\boldsymbol{k}_{\beta}\right) \cdot\left(\boldsymbol{r}_{a}-\boldsymbol{r}_{c}\right)-\left(\boldsymbol{k}_{x}-\boldsymbol{k}_{\beta}\right) \cdot \Delta \boldsymbol{r}_{c}+\left(\boldsymbol{k}_{\mathrm{P}}-\boldsymbol{k}_{\mathrm{Q}}\right) \cdot\left(\boldsymbol{s}_{x}-\boldsymbol{s}_{\beta}\right)\right\}\right]\right\rangle
\end{aligned}
$$

(The term $\left(\boldsymbol{k}_{\alpha}-\boldsymbol{k}_{\beta}\right) \cdot \Delta \boldsymbol{r}_{c}$ can be neglected)

$$
\begin{aligned}
= & N_{1}^{2} N_{2}\left(N_{2}-1\right)\left\langle\exp \left\{\mathrm{i}\left(\boldsymbol{k}_{\alpha}-\boldsymbol{k}_{\beta}\right) \cdot\left(\boldsymbol{r}_{a}-\boldsymbol{r}_{h}\right)+\mathrm{i}\left(\boldsymbol{k}_{\mathrm{P}}-\boldsymbol{k}_{\mathrm{Q}}\right) \cdot\left(\boldsymbol{s}_{\alpha}-\boldsymbol{s}_{\beta}\right)\right\}\right. \\
& \left.\times \exp \left\{-D|\tau|\left(\left|\boldsymbol{k}_{\alpha}-\boldsymbol{k}_{\mathrm{Q}}\right|^{2}+\left|\boldsymbol{k}_{\beta}-\boldsymbol{k}_{\mathrm{Q}}\right|^{2}\right)\right\}\right\rangle, \\
(2 \mathrm{II})= & \left\langle\sum _ { a , b } ^ { N _ { 1 } } \sum _ { \alpha , \beta } ^ { N _ { 2 } } \operatorname { e x p } \left[\mathrm { i } \left\{\left(\boldsymbol{k}_{0}-\boldsymbol{k}_{x}\right) \cdot \Delta \boldsymbol{r}_{a}-\left(\boldsymbol{k}_{0}-\boldsymbol{k}_{\beta}\right) \cdot \boldsymbol{r}_{b}\right.\right.\right. \\
& \left.\left.\left.+\left(\boldsymbol{k}_{x}-\boldsymbol{k}_{\mathrm{Q}}\right) \cdot \Delta \boldsymbol{s}_{\alpha}-\left(\boldsymbol{k}_{\beta}-\boldsymbol{k}_{\mathrm{Q}}\right) \cdot \Delta \boldsymbol{s}_{\beta}+\left(\boldsymbol{k}_{\mathrm{P}}-\boldsymbol{k}_{\mathrm{Q}}\right) \cdot\left(\boldsymbol{s}_{\alpha}-\boldsymbol{s}_{\beta}\right)\right\}\right]\right\rangle \\
= & N_{1}\left(N_{1}-1\right) N_{2}\left(N_{2}-1\right)\left\langle\exp \left\{\mathrm{i}\left(\boldsymbol{k}_{\mathrm{P}}-\boldsymbol{k}_{\mathrm{Q}}\right) \cdot\left(\boldsymbol{s}_{\alpha}-\boldsymbol{s}_{\beta}\right)\right\}\right. \\
& \times \exp \left\{-D|\tau|\left(\left|\boldsymbol{k}_{0}-\boldsymbol{k}_{\alpha}\right|^{2}+\left|\boldsymbol{k}_{0}-\boldsymbol{k}_{\beta}\right|^{2}\right.\right. \\
& \left.\left.\left.+\left|\boldsymbol{k}_{x}-\boldsymbol{k}_{\mathrm{Q}}\right|^{2}+\left|\boldsymbol{k}_{\beta}-\boldsymbol{k}_{\mathrm{Q}}\right|^{2}\right)\right\}\right\rangle .
\end{aligned}
$$

If we assume that $N_{1}, N_{2} \gg 1$ as before and if within $1 \%$ the next equations hold:

$$
\begin{aligned}
& \left|\boldsymbol{k}_{0}-\boldsymbol{k}_{\gamma}\right|^{2} \simeq\left|\boldsymbol{k}_{0}-\boldsymbol{k}_{1}\right|^{2}, \\
& \left|\boldsymbol{k}_{x}-\boldsymbol{k}_{\mathrm{Q}}\right|^{2}+\left|\boldsymbol{k}_{\beta}-\boldsymbol{k}_{\mathrm{O}}\right|^{2} \simeq 2\left|\boldsymbol{k}_{1}-\boldsymbol{k}_{2}\right|^{2},
\end{aligned}
$$

then we can give the final result in the following form:

$$
\begin{aligned}
\Phi_{2} & \left(\boldsymbol{R}_{\mathrm{P}}, \boldsymbol{R}_{\mathrm{Q}} ; \tau\right) \\
= & N_{1}^{2} N_{2}^{2}\left(\mu^{2}\left|E_{\mathrm{O}}\right| / R_{1} R_{2}\right)^{4} \\
& \times\left[1+\left\langle\exp \left\{i\left(\boldsymbol{k}_{\alpha}-\boldsymbol{k}_{\gamma}\right) \cdot\left(\boldsymbol{r}_{a}-\boldsymbol{r}_{b}\right)\right\}\right\rangle \exp \left(-2 D|\tau|\left|\boldsymbol{k}_{0}-\boldsymbol{k}_{1}\right|^{2}\right)\right.
\end{aligned}
$$




$$
\begin{aligned}
& +\left\langle\exp \left\{\mathrm{i}\left(\boldsymbol{k}_{\alpha}-\boldsymbol{k}_{\beta}\right) \cdot\left(\boldsymbol{r}_{a}-\boldsymbol{r}_{b}\right)+\mathrm{i}\left(\boldsymbol{k}_{\mathrm{P}}-\boldsymbol{k}_{\mathrm{Q}}\right) \cdot\left(\boldsymbol{s}_{\alpha}-\boldsymbol{s}_{\beta}\right)\right\}\right\rangle \\
& \times \exp \left(-2 D|\tau|\left|\boldsymbol{k}_{1}-\boldsymbol{k}_{2}\right|^{2}\right)+\left\langle\exp \left\{\mathrm{i}\left(\boldsymbol{k}_{\mathrm{P}}-\boldsymbol{k}_{\mathrm{Q}}\right) \cdot\left(\boldsymbol{s}_{\alpha}-\boldsymbol{s}_{\beta}\right)\right\}\right\rangle \\
& \left.\times \exp \left\{-2 D|\tau|\left(\left|\boldsymbol{k}_{0}-\boldsymbol{k}_{1}\right|^{2}+\left|\boldsymbol{k}_{1}-\boldsymbol{k}_{2}\right|^{2}\right)\right\}\right] .
\end{aligned}
$$

Inspection of eq. (46) shows that the total expression cannot be written as the product of a factor that describes only the spatial correlations and one that describes only the temporal correlations.

\subsection{Spectral noise density of the photodetector current}

The spectral densities of the optical field and of the intensity fluctuations can easily be derived from the correlation function by the use of the Wiener-Khintchine theorem ${ }^{1-3,23}$ ). The spectral noise density of the fluctuations in the photodetector current depends on the instantaneous light intensity integrated over the detector surface with area $A$. Thus:

$$
\begin{aligned}
S(f)= & 2 \eta^{2} e^{2} M^{2} \int_{-\infty}^{\infty} \mathrm{d} \tau \int_{A} \mathrm{~d} \boldsymbol{R}_{\mathrm{P}} \int_{\boldsymbol{A}} \mathrm{d} \boldsymbol{R}_{\mathrm{Q}}\left\{\Phi\left(\boldsymbol{R}_{\mathrm{P}}, \boldsymbol{R}_{\mathrm{Q}} ; \tau\right)-\langle I\rangle^{2}\right\} \\
& \times \exp (\mathrm{i} 2 \pi f \tau)+S_{0},
\end{aligned}
$$

where $\eta$ is the detection efficiency, $e$ is the electronic charge and $M$ is the mean multiplication factor of the detector. The term $S_{0}$ is due to shot noise and fluctuations in the multiplication factor and is supposed to be independent of the frequency $f$. The mean detector current is:

$$
\langle i\rangle=\eta e M A\langle I\rangle .
$$

According to eq. (9) the excess noise spectrum in case of single scattering is then found to be a Lorentz spectrum:

$$
S_{1}(f)-S_{0}=4\langle i\rangle^{2} \frac{F_{1}(A)}{A^{2}} \frac{2 D\left|k_{0}-k_{\mathrm{s}}\right|^{2}}{(2 \pi f)^{2}+\left(2 D \mid k_{0}-k_{\mathrm{s}}\right)^{2}},
$$

where $F_{1}(A)$ is the spatial coherence factor defined by:

$$
F_{1}(A)=\int_{A} \mathrm{~d} \boldsymbol{R}_{\mathrm{P}} \int_{A} \mathrm{~d} \boldsymbol{R}_{\mathrm{Q}}\left|\left\langle E_{1}^{*}\left(\boldsymbol{R}_{\mathrm{P}}, t\right) E_{1}\left(\boldsymbol{R}_{\mathrm{Q}}, t\right)\right\rangle\right|^{2} .
$$

The bandwidth of the excess noise spectrum, defined as the frequency at halfheight, is:

$$
f_{\frac{1}{2}}(\theta)=D\left|k_{0}-\boldsymbol{k}_{\mathrm{s}}\right|^{2} / \pi=4 D k_{0}^{2} \sin ^{2} \frac{1}{2} \theta / \pi,
$$

where $\theta$ is the angle between $\boldsymbol{k}_{0}$ and $\boldsymbol{k}_{\mathrm{s}}$. 
For the noise spectrum in case of double scattering we distinguish the two cases mentioned before.

Case (i), cells close together. From eq. (35) it follows that:

$$
S_{2}(f)-S_{0}=4\left\langle i_{2}\right\rangle^{2} \frac{F_{2}(A)}{A^{2}} \frac{2 D\left(\left|\boldsymbol{k}_{0}-\boldsymbol{k}_{1}\right|^{2}+\left|\boldsymbol{k}_{1}-\boldsymbol{k}_{2}\right|^{2}\right)}{(2 \pi f)^{2}+\left\{2 D\left(\left|\boldsymbol{k}_{0}-\boldsymbol{k}_{1}\right|^{2}+\left|\boldsymbol{k}_{1}-\boldsymbol{k}_{2}\right|\right)\right\}^{2}},
$$

where

$$
F_{2}(A)=\int_{\boldsymbol{A}} \mathrm{d} \boldsymbol{R}_{\mathrm{P}} \int_{\boldsymbol{A}} \mathrm{d} \boldsymbol{R}_{\mathrm{Q}}\left|\left\langle\exp \left\{\mathrm{i}\left(\boldsymbol{k}_{\mathrm{P}}-\boldsymbol{k}_{\mathrm{Q}}\right) \cdot \boldsymbol{s}_{\alpha}\right\}\right\rangle\right|^{2}
$$

As we see in eq. (52) the double scattering spectrum in excess to $S_{0}$ has a lorentzian shape with bandwidth:

$$
f_{\frac{1}{2}}\left(\theta_{1}, \theta_{2}\right)=4 D k_{0}^{2}\left(\sin ^{2} \frac{1}{2} \theta_{1}+\sin ^{2} \frac{1}{2} \theta_{2}\right) / \pi=f_{\frac{1}{2}}\left(\theta_{1}\right)+f_{\frac{1}{2}}\left(\theta_{2}\right) .
$$

Case (ii), cells far apart. Eqs. (46) and (47) yield:

$$
\begin{aligned}
S_{2}(f)-S_{0}= & 4\left\langle i_{2}\right\rangle^{2}\left[B_{1} \frac{2 D\left|\boldsymbol{k}_{0}-\boldsymbol{k}_{1}\right|^{2}}{(2 \pi f)^{2}+\left(2 D\left|\boldsymbol{k}_{0}-\boldsymbol{k}_{1}\right|^{2}\right)^{2}}\right. \\
& +B_{2} \frac{2 D\left|\boldsymbol{k}_{1}-\boldsymbol{k}_{2}\right|^{2}}{(2 \pi f)^{2}+\left(2 D\left|\boldsymbol{k}_{1}-\boldsymbol{k}_{2}\right|^{2}\right)^{2}} \\
& \left.+B_{3} \frac{2 D\left(\left|\boldsymbol{k}_{0}-\boldsymbol{k}_{1}\right|^{2}+\left|\boldsymbol{k}_{1}-\boldsymbol{k}_{2}\right|^{2}\right)}{(2 \pi f)^{2}+\left\{2 D\left(\left|\boldsymbol{k}_{0}-\boldsymbol{k}_{1}\right|^{2}+\left|\boldsymbol{k}_{1}-\boldsymbol{k}_{2}\right|^{2}\right)\right\}^{2}}\right],
\end{aligned}
$$

where

$$
\begin{aligned}
B_{1}= & \left\langle\exp \left\{\mathrm{i}\left(\boldsymbol{k}_{x}-\boldsymbol{k}_{\gamma}\right) \cdot\left(\boldsymbol{r}_{a}-\boldsymbol{r}_{b}\right)\right\}\right\rangle, \\
B_{2}= & \left(1 / A^{2}\right) \int_{\boldsymbol{A}} \mathrm{d} \boldsymbol{R}_{\mathrm{P}} \int_{\boldsymbol{A}} \mathrm{d} \boldsymbol{R}_{\mathrm{Q}}\left\langle\operatorname { e x p } \left\{\mathrm{i}\left(\boldsymbol{k}_{\alpha}-\boldsymbol{k}_{\beta}\right) \cdot\left(\boldsymbol{r}_{a}-\boldsymbol{r}_{b}\right)\right.\right. \\
& \left.\left.+\mathrm{i}\left(\boldsymbol{k}_{\mathrm{P}}-\boldsymbol{k}_{\mathrm{Q}}\right) \cdot\left(\boldsymbol{s}_{\alpha}-\boldsymbol{s}_{\beta}\right)\right\}\right\rangle . \\
B_{3}= & \left(1 / A^{2}\right) \int_{\boldsymbol{A}} \mathrm{d} \boldsymbol{R}_{\mathrm{P}} \int_{\boldsymbol{A}} \mathrm{d} \boldsymbol{R}_{\mathrm{Q}}\left\langle\exp \left\{\mathrm{i}\left(\boldsymbol{k}_{\mathrm{P}}-\boldsymbol{k}_{\mathrm{Q}}\right) \cdot\left(\boldsymbol{s}_{x}-\boldsymbol{s}_{\beta}\right)\right\}\right\rangle .
\end{aligned}
$$

The excess noise spectrum consists now of three terms, each of which is the product of a spatial coherence factor and a Lorentz spectrum. Note that $B_{3}=F_{2}(A) / A^{2}$. 


\section{Experimental set-up and results}

In the double scattering experiment we used two lasers: a three mode $\mathrm{HeNe}$ laser (Spectra Physics model 120) with wavelength $\lambda=632.8 \mathrm{~nm}$ and a power of $5 \mathrm{~mW}$ and a single mode $\mathrm{Kr}$-ion laser (Spectra Physics model 165) with wavelength $\lambda=647.1 \mathrm{~nm}$ and a power adjustable between 40 and $100 \mathrm{~mW}$. The scattering medium consisted of Latex spheres suspended in water. In every scattering experiment both cells were filled with the same suspension. We found no difference in the interpretation of the experimental results when the spheres had a diameter of $101 \mathrm{~nm}$ or $332 \mathrm{~nm}$. So we restrict ourselves to a report of the measurements where we used spheres with diameter $332 \mathrm{~nm}$. We note that the particle size was of the same order of magnitude as the wavelength of the light. So the criterion for Rayleigh scattering $2 \varrho \ll \lambda$ was not satisfied. Nevertheless the frequency dependence of the single scattering spectrum is still given by eq. $\left.(49)^{2,8}\right)$.

Between the two cells and between the second cell and the detector diaphragms were placed (see fig. 1) in order to limit the solid angles of the light beams. We checked carefully that only light coming from the second cell was seen by the photodetector. Polarization analyzers, inserted in the light beams with axes parallel to the polarization of the incident laser light, did not change the frequency dependence of the measured noise spectra. The light signal after double scattering was always very weak. Normally detector currents were about $0.5 \mu \mathrm{A}$. In order to enhance the detection efficiency of the photomultiplier (EMI 9558A) we stuck a small prism with immersion oil on the window of the detector ${ }^{24}$ ). When the two cells were close together we reduced the light losses caused by reflections by sticking the two cells on either side of a glass plate. The spectral noise density of the photocurrent was measured by means of a wave analyzer (Bruel \& Kjaer type 2114).

We do not know the concentration of the particles in the suspensions we have used. In order to be sure that in each cell only single scattering occurred we measured the homodyne spectrum of the light scattered by the first cell. The experimental results of two of these measurements can be found in fig. 3 where the experimental data (the dots) are compared with Lorentz spectra (full curves). Experimentally we found the bandwidths: $f_{\frac{1}{2}}\left(\theta_{1}=60^{\circ}\right)=86 \mathrm{~Hz}$ and $f_{\frac{1}{2}}\left(\theta_{1}=90^{\circ}\right)$ $=165 \mathrm{~Hz}$. According to eq. (51) the theory predicts the bandwidths: $f_{\frac{1}{2}}\left(\theta_{1}=60^{\circ}\right)$ $=85 \mathrm{~Hz}$ and $f_{\frac{1}{2}}\left(\theta_{1}=90^{\circ}\right)=169 \mathrm{~Hz}$ for particles with diameter $2 o=332 \mathrm{~nm}$ at a wavelength of $\lambda=647.1 \mathrm{~nm}$ and a temperature $T=28^{\circ} \mathrm{C}$. As the error in the experimental determination of the bandwidths in all our experiments was about $4 \%$ and the experimental data corresponded to Lorentz spectra over almost five decades we may assume that in spite of possible high particle concentration only single scattering prevails in each cell. 


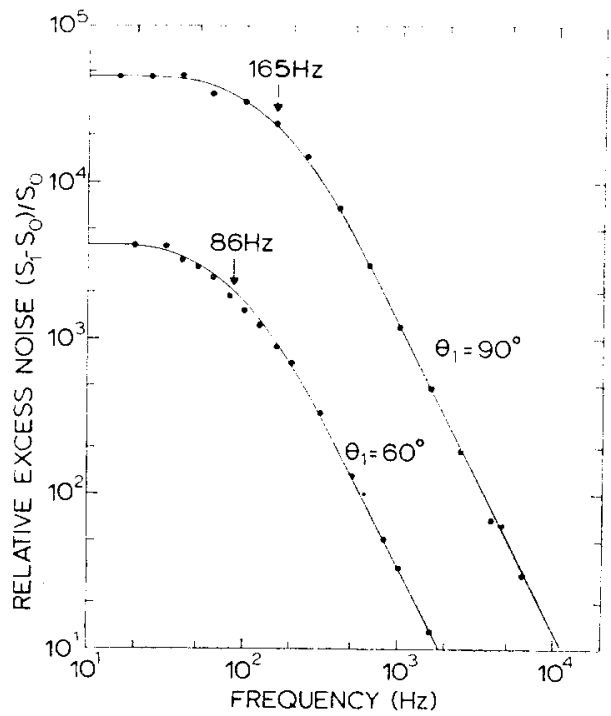

Fig. 3

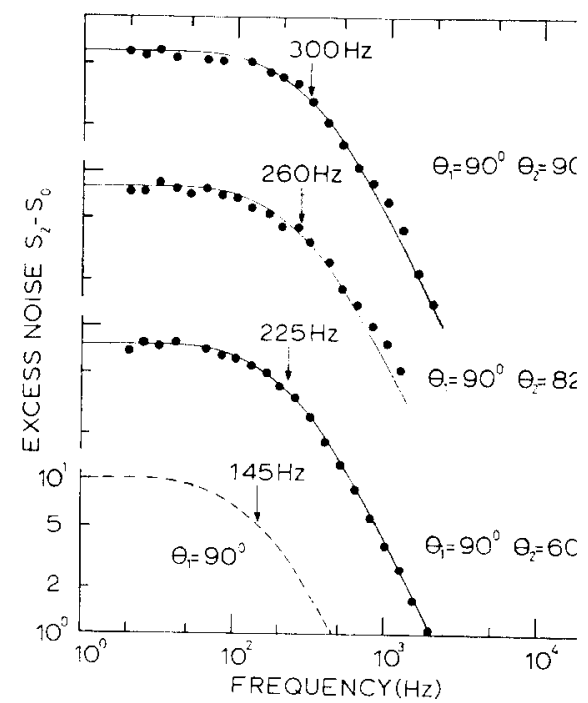

Fig. 4

Fig. 3. Relative excess noise $\left(S_{1}-S_{0}\right) / S_{0}$ versus frequency of singly scattered light at an $\theta_{1}=90^{\circ}$ and $\theta_{2}=60^{\circ}$. The dots are the experimental results, the full curves are Lorentz sper and the arrows indicate the bandwidth of the spectra.

Fig. 4. Double logarithmic plot of $S_{2}-S_{0}$ (in arbitrary units) of doubly scattered light ve frequency. The distance between the scattering cells was $1.5 \times 10^{-2} \mathrm{~m}$. The first scattering as was $\theta_{1}=90^{\circ}$ and the second scattering angles were $\theta_{2}=90^{\circ}, 82^{\circ}, 60^{\circ}$. The experimental res (the dots) are compared by Lorentz spectra (full curves). The dashed curve gives the freque dependence of the noise in singly scattered light at a scattering angle $\theta_{1}=90^{\circ}$.

Case (i), cells close together. Characteristic values for the distances in this expe ment were: $R_{1}=1.5 \times 10^{-2} \mathrm{~m}$ and $R_{2}=4.2 \times 10^{-1} \mathrm{~m}$. The effective diame of the photodetector was $5 \times 10^{-3} \mathrm{~m}$. If we represent the scattering volumes : proximately by cuboids, the first scattering volume has the dimensions: $1 \times 10$ by $1 \times 10^{-4}$ by $2 \times 10^{-3} \mathrm{~m}$ and the second scattering volume had the dimensior $2 \times 10^{-3}$ by $2 \times 10^{-3}$ by $2 \times 10^{-3} \mathrm{~m}$.

Fig. 4 gives three excess noise spectra (the dots) measured on doubly scatter $\mathrm{He}-\mathrm{Ne}$ laser light at particles with diameter $2 \varrho=332 \mathrm{~nm}$ and at a temperat $T=21.5^{\circ} \mathrm{C}$. Lorentz spectra could be fitted well to the experimental points shown by the full curves. The first scattering angle was in all cases $\theta_{1}=90^{\circ} \mathrm{b}$ the second scattering angle was varied as indicated in the figure. The dashed cur shows the theoretical frequency dependence of singly scattered light at a scatteri angle $\theta_{1}=90^{\circ}$. The bandwidths of the double scattering spectra (indicated the arrows) depart markedly from the bandwidth of the single scattering spe trum. We compare the experimentally obtained values for the bandwidths of $t$ 
double scattering spectra with the bandwidths calculated from eq. (54) (within the brackets):

$$
\begin{aligned}
& f_{\frac{1}{2}}\left(\theta_{1}=90^{\circ}, \theta_{2}=90^{\circ}\right)=300 \mathrm{~Hz}(290 \mathrm{~Hz}), \\
& f_{\frac{1}{2}}\left(\theta_{1}=90^{\circ}, \theta_{2}=82^{\circ}\right)=260 \mathrm{~Hz}(270 \mathrm{~Hz}) \text { and } \\
& f_{\frac{1}{2}}\left(\theta_{1}=90^{\circ}, \theta_{2}=60^{\circ}\right)=225 \mathrm{~Hz}(218 \mathrm{~Hz}) .
\end{aligned}
$$

Within the experimental error, which was about $4 \%$, agreement between experimentally determined and theoretically calculated bandwidths is found.

Case (ii), cells far apart. Characteristic values of the distances in the experimental set-up were now: $R_{1}=5.0 \times 10^{-1} \mathrm{~m}$ and $R_{2}=3.0 \times 10^{-1} \mathrm{~m}$. The effective diameter of the detector was $5 \times 10^{-2} \mathrm{~m}$. The first scattering volume represented approximately as a cuboid, had the dimensions $1 \times 10^{-4}$ by $1 \times 10^{-4}$ by $1 \times 10^{-3} \mathrm{~m}$ and the dimensions of the second scattering volume were $1 \times 10^{-2}$ by $1 \times 10^{-2}$ by $1 \times 10^{-2} \mathrm{~m}$. In fig. 5 three spectra (the dots) are shown measured on laser light $(\lambda=647.1 \mathrm{~nm})$ doubly scattered by particles $(2 o=332 \mathrm{~nm})$ at a temperature

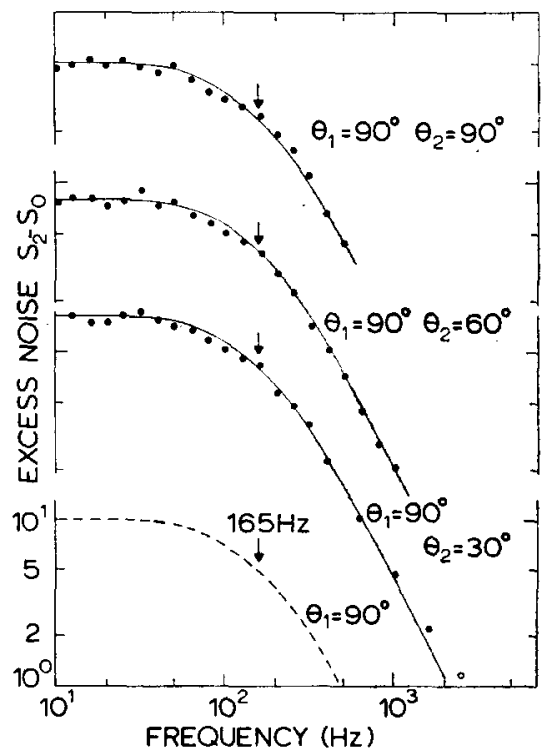

Fig. 5. Excess noise spectra $S_{2}-S_{0}$ in arbitrary units of doubly scattered light. The cells were at a distance of $5.0 \times 10^{-1} \mathrm{~m}$ from each other. The first scattering angle was $\theta_{1}=90^{\circ}$, the second scattering angles were $\theta_{2}=90^{\circ}, 60^{\circ}, 30^{\circ}$. The full curves are Lorentz spectra fitted on the experimental points. The dashed curve gives the frequency dependence of the spectra for single scattering at a scattering angle $\theta_{1}=90^{\circ}$. 
$T=28^{\circ} \mathrm{C}$. The experimental points are well matched by Lorentz spectra (full curves). The dashed Lorentz spectrum gives the frequency dependence of the noise spectral density of singly scattered light at a scattering angle $\theta_{1}=90^{\circ}$ (see fig. 3). In the double scattering experiment the first scattering angle was $\theta_{1}=90^{\circ}$ and the second angle $\theta_{2}$ was varied. However, as the arrows in fig. 5 indicate, all bandwidths of the double scattering spectra were the same. The bandwidths were (within the experimental error of $4 \%$ ) equal to the spectral bandwidth of the light after the first scattering process: $f_{\frac{1}{2}}\left(\theta_{1}=90^{\circ}\right)=165 \mathrm{~Hz}$.

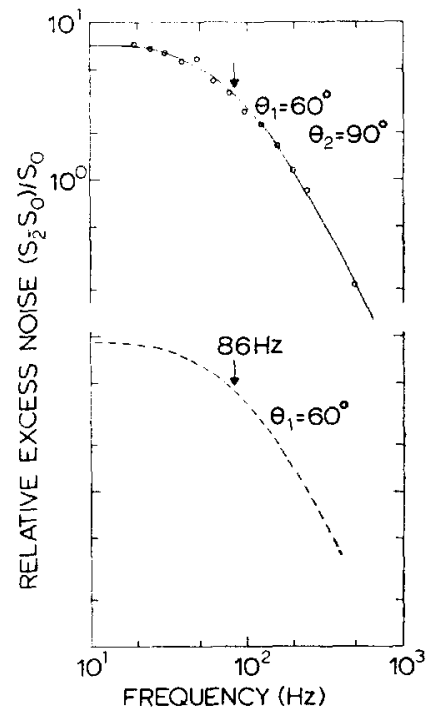

Fig. 6. Relative excess noise $\left(S_{2}-S_{0}\right) / S_{0}$ of doubly scattered light when the distance between the cells was $5.0 \times 10^{-1} \mathrm{~m}$. The first scattering angle is $\theta_{1}=60^{\circ}$ and the second one $\theta_{2}=90^{\circ}$. The frequency dependence of the spectrum for single scattering at $\theta_{1}=60^{\circ}$ is given by the dashed curve.

If we changed the first scattering angle, however, to $\theta_{1}=60^{\circ}$ the bandwidth of the double scattering spectrum was changed into $f_{\frac{1}{2}}\left(\theta_{1}=60^{\circ}, \theta_{2}=90^{\circ}\right)$ $=86 \mathrm{~Hz}$ as can be seen in fig. 6 . And this latter bandwidth is again equal to the spectral bandwidth of the intensity fluctuations of singly scattered light at an angle $\theta_{1}=60^{\circ}$.

\section{Discussion}

Good agreement between theory and experiment was found when the two scattering cells were close together (case (i)). In the derivation of the intensity cross- 
correlation function for this case the assumption given in eq. (30) was used. From the experimental geometry described in sec. 3 it follows that within $1 \%$

$$
\left|\boldsymbol{k}_{0}-\boldsymbol{k}_{a \alpha}\right|^{2}+\left|\boldsymbol{k}_{a \alpha}-\boldsymbol{k}_{\mathrm{Q}}\right|^{2} \simeq\left|\boldsymbol{k}_{0}-\boldsymbol{k}_{1}\right|^{2}+\left|\boldsymbol{k}_{1}-\boldsymbol{k}_{2}\right|^{2}
$$

So the use of eq. (30) is justified.

In the second case we studied, the scattering cells were far apart (case (ii)). From the experimental geometry as mentioned in sec. 3 it follows that eq. (45) holds. In sec. 3 we found that the frequency dependence of the double scattering spectra was the same as that of the single scattered spectrum measured at the first scattering angle. This experimental result can be explained with the help of eq. (55) as a rough calculation of the spatial coherence factors $B_{1}, B_{2}$ and $B_{3}$ gives:

$$
B_{2} / B_{1} \simeq 10^{-4} \quad \text { and } \quad B_{3} / B_{1} \simeq 10^{-4}
$$

So the first term in eq. (55) dominates and this term has the same frequency dependence as the spectrum of the singly scattered radiation.

Inspection of eq. (56) makes clear the meaning of the spatial coherence factors $B_{1}, B_{2}$ and $B_{3}$. The term $B_{1}$ describes the spatial coherence of the light beam in the second cell, whereas $B_{3}$ describes the spatial coherence at the detector surface. The term $B_{2}$ depends on the spatial coherence of the light beam in the second cell as well as on the spatial coherence of the light at the detector surface. The detector area was about a factor $10^{6}$ larger than the "coherence area"1-3) associated with the second cell, whereas the cross section of the beam in the second cell was about a factor $10^{2}$ larger than the coherence area associated with the first cell. Therefore the fluctuations in the light induced by the random movements of the scatterers in the first cell dominate those induced by the scatterers in the second cell. The solid angle within which the light is scattered from the first to the second scattering cell was made small enough to ensure a beam with well-defined properties; however, light scattered from the second cell to the detector was emitted within a large solid angle, so that we had a detectable signal.

Next we compare our theoretical results with those of other authors ${ }^{15-17}$ ), Ivanov $e^{t} a l .{ }^{15}$ ) have calculated the autocorrelation function of the intensity of doubly scattered radiation. Their scattering medium was a layer with moving centres and can be best compared with our case (i) where the cells were close together. Ivanov et al. did not take into account the spatial correlation across the detector surface. If we put $\boldsymbol{k}_{\mathrm{P}}=\boldsymbol{k}_{\mathrm{Q}}=\boldsymbol{k}_{2}$ in eq. (34) we find agreement with their result given in eq. (11) of ref. 15. But where we have simplified eq. (34) to eq. (35), Ivanov et al. have performed an integration over the scattering volumes. Their numerical calculation shows that in fairly good approximation the autocorrelation function $\Phi_{2}\left(\boldsymbol{R}_{\mathrm{P}}, \boldsymbol{R}_{\mathrm{P}} ; \tau\right)-\langle I\rangle^{2}$ is an exponential function. They have measured the spectral bandwidth of the intensity fluctuations as a function of concen- 
tration of the particles. But their results cannot be compared with ours because they detected the light from single, double and multiple scattering simultaneously, whereas we detected doubly scattered light only. Moreover they assumed erroneously that singly and doubly scattered light are statistically independent. In a study of multiple scattering Kelly ${ }^{16}$ ) has calculated the cross-correlation between singly and doubly scattered light. This correlation is not present in our analysis because, as stated before, we investigated double scattering only. Correlation functions for purely doubly scattered light were not given in Kelly's paper.

Bertolotti et al. ${ }^{17}$ ) have calculated the photon counting distribution of gaussian light scattered by a gaussian medium. If the medium consisted of randomly moving particles they found for the second factorial moment of the photon counts:

$$
\langle n(n-1)\rangle=4\langle n\rangle^{2},
$$

where $n$ is the number of photons counted in a time-interval of length $\tau$, which was much shorter than the coherence time of the radiation. This scattering process can be compared with our case (ii) where the cells were far apart, since we may assume that the singly scattered field is gaussian $\left.{ }^{1-3,10,13}\right)$.

The relation between intensity fluctuations and $\langle n(n-1)\rangle$ is generally given $b^{25}$ ) ( $A$ less than the coherence area):

$$
(\eta A \tau)^{2}\left\langle I^{2}\right\rangle=\langle n(n-1)\rangle .
$$

The value of $(\eta A \tau)^{2}\left\langle I^{2}\right\rangle$ can be derived from the noise spectral density of the photocurrent with the help of the relation:

$$
(\eta A \tau)^{2}\left\langle I^{2}\right\rangle=(\tau / e M)^{2} \int_{0}^{x}\left\{S(f)-S_{0}\right\} \mathrm{d} f+(\eta A \tau\langle I\rangle)^{2},
$$

where $\eta A \tau\langle I\rangle=\langle n\rangle$. Bertolotti et al. did not take into account the effect of spatial coherence, so we take $B_{1}=B_{2}=B_{3}=1$ in eq. (55). Eqs. (48), (55) and (59) now yield:

$$
(\eta A \tau)^{2}\left\langle I^{2}\right\rangle=3(\eta A \tau\langle I\rangle)^{2}+\langle n\rangle^{2}=4\langle n\rangle^{2},
$$

which is in agreement with eq. (57).

Finally we give some thought to the question whether the doubly scattered light is gaussian or not. In case (i) the intensity correlation function obeys eq. (36). And this is a necessary but not a sufficient condition for a gaussian field. In case (ii), however, eq. (36) is not satisfied as inspection of eqs. (41) and (46) makes clear. So in case (ii) the doubly scattered field is certainly not gaussian. This can be explained as follows. If we accept the widely used assumption that the singly scattered field is gaussian, we can interpret the double scattered field as a product 
of two gaussian variables. But a product of two gaussian variables never results in a new gaussian variable. Kelly ${ }^{16}$ ) has concluded that an $n$-fold scattered field does not obey gaussian statistics. But he found that $n$ must be quite large before the departure from gaussian statistics has any experimental significance. His conclusion seems to be correct in the case when the cells are close together but is certainly untrue when the cells are far apart. The non-gaussian character of the light in the latter case is confirmed by the experiments. For, if $E_{2}\left(\boldsymbol{R}_{\mathrm{P}}, t\right)$ was gaussian, only the third term on the right of eq. (55) would have been found. However, this term in the noise spectral density has not been observed at all in the spectra measured.

Having studied the fluctuations in doubly scattered laser light experimentally and theoretically in this paper, we have come to the following conclusions: in the analysis of double scattering spectra the geometry of the experimental set-up is very important and separation of temporal and spatial correlations cannot always be obtained. In addition we found that a doubly scattered field can have nongaussian statistics.

\section{Acknowledgements}

The authors are grateful to Professor C.Th.J.Alkemade and to Dr. R.J.J. Zijlstra for many interesting discussions and suggestions in the course of this investigation. The technical advice of Mr. F. Wollenberg is greatly appreciated.

\section{Appendix}

From eq. (27) it follows that for a displacement $\Delta r$ in the time interval $\tau$ :

$$
\left\langle\left(\Delta r_{\tau}\right)^{2}\right\rangle=6 D \tau .
$$

According to eqs. (3) and (5) the correlation time of the singly scattered field is:

$$
t_{\mathrm{c}}=1 / D\left|\boldsymbol{k}_{0}-\boldsymbol{k}_{\mathrm{s}}\right|^{2}=\lambda^{2} / D\left(4 \pi n \sin \frac{1}{2} \theta\right)^{2},
$$

where $\lambda$ is the wavelength of the laser light in vacuum, $n$ is the refractive index of the solvent and $\theta$ is the angle between $\boldsymbol{k}_{0}$ and $\boldsymbol{k}_{\mathrm{s}}$. If we neglect scattering in a forward direction where $\theta$ is small, we see that:

$$
\left\langle\left(\Delta r_{t_{c}}\right)^{2}\right\rangle^{\frac{1}{2}} \simeq \lambda .
$$




\section{References}

1) G.B.Benedek, in: Polarisation, Matière et Rayonnement, Livre de Jubilé en l'honneur de Professeur A. Kastler (Presses Universitaires de France, Paris, 1969) p. 49

2) H.Z.Cummins and H.L. Swinney, in: Progress in Optics, vol.8, E. Wolf, ed. (North-Holland, Amsterdam, 1970) p. 133.

3) B. Chu, Laser Light Scattering (Academic Press, New York, 1974).

4) R.Pecora, J. Chem. Phys. 40 (1964) 1604.

5) F.T.Arecchi, M. Giglio and U.Tartari, Phys. Rev. 163 (1967) 186.

6) R. Foord, E.Jakeman, C.J.Oliver, R.J.Blagrove, E. Wood and A. R. Peacocke, Nature 227 (1970) 242.

7) N.A.Clark, J.H.Lunacek and G.B.Benedek, Am. J. Phys. 38 (1970) 575.

8) K. Ohbayashi, S. Kagoshima and A.Ikushima, Japan. J. Appl. Phys. 11 (1972) 808

9) D.W. Schaefer and B. J. Berne, Phys. Rev. Letters 28 (1972) 475.

10) S.H.Chen and P. Tartaglia, Opt. Commun. 6 (1972) 119.

11) P. Tartaglia and S.H.Chen, Opt. Commun. 7 (1973) 379.

12) T. Aoki, Y. Okabe and K.Sakurai, Phys. Rev. A10 (1974) 259.

13) P. N. Pusey, D.W. Schaefer and D.E Koppel, J. Phys. A: Gen. Phys. 7 (1974) 530.

14) D.W.Schaefer and P.N.Pusey, Phys. Rev. Letters 29 (1972) 843.

15) A.P.Ivanov, A. Ya. Khairullina and A.P.Chaikovskii, Opt. Spectrose. 35 (1973) 668.

16) H.C. Kelly, J. Phys. A: Gen. Phys. 6 (1973) 353.

17) M. Bertolotti, B. Crosignani and P. di Porto, J. Phys. A: Gen. Phys. 3 (1970) L37.

18) C. Kittel, Elementary Statistical Physics (John Wiley, New York, 1964).

19) H.C. van der Hulst, Light Scattering by Small Particles (John Wiley, New York, 1957).

20) E.Jakeman, C.J. Oliver and E.R.Pike, J. Phys. A: Gen. Phys. 3 (1970) L45.

21) L. Mandel, in: Progress in Optics, vol.2, E. Wolf, ed. (North-Holland, Amsterdam, 1970) p. 181.

22) S. Chandrasekhar, Rev. Mod. Phys. 15 (1943) 1.

23) L. Mandel and E.Wolf, Rev. Mod. Phys. 37 (1965) 231.

24) W.D.Gunter, G.R.Grant and S. A.Shaw, Appl. Opt. 9 (1970) 251.

25) C.L. Mehta, in: Progress in Optics, vol. 8, E.Wolf, ed. (North-Holland, Amsterdam, 1970) p. 373. 